\title{
Semi-Nonparametric Estimation of Consumer Search Costs *
}

\author{
José Luis Moraga-González ${ }^{\dagger}$ \\ Zsolt Sándor ${ }^{\ddagger}$ \\ Matthijs R. Wildenbeest $\S$
}

June 2010

\begin{abstract}
This paper studies the estimation of the cost of non-sequential search. We provide a new method based on semi-nonparametric (SNP) estimation that allows us to pool price data from different consumer markets with the same underlying search cost distribution but different valuations or selling costs. We show that pooling data from different markets increases the number of estimated critical search cost cutoffs at all quantiles of the search cost distribution, which increases the precision of the estimates. A Monte Carlo study shows that the method works well in small samples. We apply our method to a data set of online prices for memory chips and find that the search cost density is essentially bimodal such that a large fraction of consumers searches very little, while a smaller fraction of consumers samples a relatively large number of stores.
\end{abstract}

Keywords: consumer search, oligopoly, search costs, semi-nonparametric estimation

JEL Classification: C14, D43, D83, L13

\footnotetext{
*Earlier drafts were circulated under the title "Nonparametric Estimation of the Costs of Non-Sequential Search." We are indebted to Vladimir Karamychev, Allard van der Made, Paulo K. Monteiro, Martin Pesendorfer, Michael Rauh, and Matthew Shum for their useful comments. The paper has also benefited from presentations at Universidad Carlos III de Madrid, University of Chicago, CORE, University of Essex, Tinbergen Institute, the ESEM Meetings 2006 (Vienna), the EEA Meetings 2007 (Budapest), and the IIOC 2009 (Boston). Financial support from the Netherlands Organization for Scientific Research (NWO) and from Marie Curie Excellence Grant MEXT-CT-2006042471 is gratefully acknowledged.

${ }^{\dagger}$ ICREA, IESE Business School and University of Groningen, E-mail: jose.1.moraga@gmail.com.

¥University of Groningen, E-mail: z.sandor@rug.nl.

${ }^{\S}$ Kelley School of Business, Indiana University, E-mail: mwildenb@indiana.edu.
} 


\section{Introduction}

A significant body of work in the economics and marketing literature has shown that search costs have far-reaching effects in economic activity. Well-known facts are that search costs alone can lead to price dispersion (Varian, 1980; Burdett and Judd, 1983; Stahl, 1989) as well as to wage and technology dispersion (Burdett and Mortensen, 1998; Acemoglu and Shimer, 2000). Search costs can also generate excessive product diversity in differentiated product markets (Anderson and Renault, 2000; Wolinsky, 1984) as well as inefficient quality investments (Wolinsky, 2005). The existence of search costs can also explain asymmetric price-cost adjustments (Tappata, 2009), price differences between online and traditional markets (Bakos, 1997; Lal and Sarvary, 1999; Brynjolfsson and Smith, 2000), and the emergence of different price institutions (Bester, 1994). ${ }^{1}$

Given the importance of the costs of search in shaping economic outcomes, part of the recent research in the area is focusing on developing techniques to estimate consumer search costs. Several estimation procedures have been proposed. Hong and Shum (2006) were the first to develop a structural method to retrieve information on consumer search costs using market data. They focus on markets for homogeneous goods and present various approaches to estimate non-sequential and sequential consumer search models using only price data. Moraga-González and Wildenbeest (2008) present an alternative estimator based on maximum likelihood for non-sequential consumer search models. Hortaçsu and Syverson (2004) and Wildenbeest (2009) study search models where search frictions coexist with vertical product differentiation. Kim, Albuquerque, and Bronnenberg (2010) estimate a more general demand model using micro-level data on search behavior to estimate consumer preferences and search costs. In all these models, consumer search costs are found to be sizable.

The present paper adds to this literature in two ways. First, we provide a proof that the critical search costs estimated by Hong and Shum (2006) and Moraga-González and Wildenbeest (2008) are indeed non-parametrically identified. Second, we provide a new method based on seminonparametric (SNP) estimation that allows us to pool price data from different consumer markets with the same underlying search cost distribution but different valuations or selling costs. Pooling data from different markets increases the number of estimated critical search cost cutoffs at all quantiles of the search cost distribution, which increases the precision of the estimates. This new method outperforms the spline approximation methods employed earlier in the literature (cf. Hong

\footnotetext{
${ }^{1}$ For a recent overview of studies on search and price dispersion, see Baye, Morgan, and Scholten (2006).
} 
and Shum, 2006; Hortaçsu and Syverson, 2004; and Moraga-González and Wildenbeest, 2008) because instead of estimating the parameters of the price distribution market by market, which ignores the link between the different data sets, our semi-nonparametric approach estimates the search cost density using all the data at a time. SNP density estimators use a flexible polynomialtype parametric function that can approximate arbitrarily closely a large class of sufficiently smooth density functions (Gallant and Nychka, 1987), which means we obtain an essentially nonparametric estimator of the search cost distribution common to all the markets. A Monte Carlo study illustrates that the estimators performs well in small samples.

To illustrate how our method works with real-world data we apply the SNP estimation procedure to a data set of online prices for ten notebook memory chips. Our estimate of the search cost distribution shows that consumers have either quite high or quite low search costs. ${ }^{2}$ Consumers with high search costs do not compare prices and this gives substantial market power to the firms; as a result, estimated price-cost margins are significantly larger than what one would expect on the basis of the observed large number of firms operating in each market.

The structure of the paper is as follows. In the next section we review the non-sequential consumer search model. The identification result, the SNP estimation method, and a Monte Carlo study are presented in Section 3. In Section 4 we estimate the search cost distribution underlying price data from ten online markets for memory chips. Finally, Section 5 concludes. Our proofs are placed in the Appendix to ease the reading.

\section{The model}

The model, proposed by Hong and Shum (2006), generalizes the non-sequential consumer search model of Burdett and Judd (1983) by adding search cost heterogeneity. ${ }^{3}$ There is a large number of firms producing a good at constant returns to scale. Their identical unit cost is equal to $r$. There is a unit mass of buyers. Each consumer wishes to purchase a single unit of the good at most. We assume that the maximum price any buyer is willing to pay for the good is $v$. Consumers must engage in costly search to observe prices. Assume they search non-sequentially and that the first price quotation is obtained at no cost. Once a consumer has observed the desired number of prices, she chooses to buy from the store charging the lowest price. We assume that consumers differ in their search costs. A buyer's search cost is drawn independently from a common atomless

\footnotetext{
${ }^{2}$ A similar finding has already been reported in earlier work (cf. Moraga-González and Wildenbeest, 2008) so it is encouraging to see that it depends neither on the estimation method nor on the data set.

${ }^{3}$ Janssen and Moraga-González (2004) studied the same model with a two-point search cost distribution.
} 
distribution $G(c)$ with support $(0, \infty)$ and positive density $g(c)$ everywhere. A consumer with search cost $c$ sampling $k$ firms incurs a total search cost $k c$. We shall denote by $K \geq 2$ the maximum number of prices consumers can observe in this market; like Hong and Shum (2006) we allow $K$ to be equal to infinity.

Firms and buyers play a simultaneous moves game. An individual firm chooses its price taking price choices of the rivals as well as consumers' search behavior as given. A firm $i$ 's strategy is denoted by a distribution of prices $F_{i}$. Let $F_{-i}$ denote the vector of strategies used by firms other than $i$. The (expected) profit to firm $i$ from charging price $p_{i}$ given rivals' strategies is denoted $\Pi_{i}\left(p_{i}, F_{-i}\right)$. Likewise, an individual buyer takes as given firm pricing and decides on his/her optimal search strategy to maximize his/her expected utility. The strategy of a consumer with search cost $c$ is then a number $k$ of prices to sample. Let the fraction of consumers sampling $k$ firms be denoted by $\mu_{k}$. We shall concentrate on symmetric Nash equilibria, i.e. equilibria where $F_{i}=F$ for all $i$. A symmetric equilibrium is a distribution of prices $F$ and a collection $\left\{\mu_{k}\right\}_{k=1}^{K}$ such that (a) $\Pi_{i}(p ; F)$ is equal to a constant $\bar{\Pi}$ for all $p$ in the support of $F, \forall i$; (b) $\Pi_{i}(p ; F) \leq \bar{\Pi}$ for all $p, \forall i$; (c) a consumer sampling $k$ firms obtains no lower utility than by sampling any other number of firms; and (d) $\sum_{k=1}^{K} \mu_{k}=1$. Let us denote the equilibrium density of prices by $f$, with maximum price $\bar{p}$ and minimum price $\underline{p}$.

Given firm behavior, ${ }^{4}$ the number of prices $k(c)$ a consumer with search cost $c$ observes must be optimal, i.e.,

$$
k(c)=\arg \min _{k} c(k-1)+\int_{\underline{p}}^{v} k p(1-F(p))^{k-1} f(p) d p .
$$

Since $k(c)$ must be an integer, the problem in equation (1) induces a partition of the set of consumers into $\left\{\mu_{k}\right\}_{k=1}^{K}$. This partition is calculated as follows. Let $E p_{1: k}$ be the expected minimum price in a sample of $k$ prices drawn from the price distribution $F$. The number

$$
c_{k}=E p_{1: k}-E p_{1: k+1}, k=1,2, \ldots, K-1
$$

is the search cost of the consumer indifferent between sampling $k$ prices and sampling $k+1$ prices. Since $c_{k}$ is a decreasing function of $k,{ }^{5}$ the fractions of consumers $\mu_{k}$ sampling $k$ prices are given

\footnotetext{
${ }^{4}$ It will become clear later that the upper bound of the price distribution must be equal to the consumer valuation.

${ }^{5}$ The cutoffs $c_{k}=E p_{1: k}-E p_{1: k+1}$ are in fact strictly monotonically decreasing in $k$ because $E p_{1: k}$ is strictly convex in $k$. A proof of this is available from the authors upon request. See also Stigler (1961), who mentions this property.
} 


$$
\begin{aligned}
& \mu_{1}=1-G\left(c_{1}\right) \\
& \mu_{k}=G\left(c_{k-1}\right)-G\left(c_{k}\right), k=2,3, \ldots, K .
\end{aligned}
$$

Given consumers' search behavior it is indeed optimal for firms to mix in prices. The upper bound of the price distribution must be $v$; this is because a firm that charges the upper bound sells only to the consumers who do not compare prices, i.e. consumers in $\mu_{1}$, and these consumers would also accept $v$. The equilibrium price distribution follows from the indifference condition that a firm should obtain the same level of profits from charging any price in the support of $F$, i.e.,

$$
(p-r)\left[\sum_{k=1}^{K} k \mu_{k}(1-F(p))^{k-1}\right]=\mu_{1}(v-r)
$$

for all prices $p$ in the support of $F$. From equation (4) it follows that the minimum price charged in the market is

$$
\underline{p}=\frac{\mu_{1}(v-r)}{\sum_{k=1}^{K} k \mu_{k}}+r
$$

\section{Statistical analysis}

\subsection{Introduction}

The econometric problem is to estimate the search cost distribution using price data. Hong and Shum (2006) and Moraga-González and Wildenbeest (2008) propose different methods that exploit equations (2) to (5) to estimate the search cost CDF. In what follows, we briefly explain the two methods proposed so far (for details see their original contributions).

Hong and Shum (2006) formulate the estimation of the unknown search cost distribution as a two-step procedure. They propose to first estimate the parameters $\left\{\mu_{k}\right\}_{k=1}^{K}$ of the equilibrium price distribution obtained from equation (4) by maximum empirical likelihood (MEL), and then to recover the collection of cutoffs in equation (2) using the empirical CDF of prices. Suppose we have a (large) data set with $n$ prices and suppose $K(\leq n-1)$ is the maximum number of prices a consumer may observe in the market. Let us assume each price $p_{j}$ has probability $\pi_{j}$. Using equilibrium condition (4), for each price $p_{i}$ we have the approximate equality

$$
\left(p_{i}-r\right)\left[\sum_{k=1}^{K} k \mu_{k}\left(1-\left[\sum_{j=1}^{n} \pi_{j} \mathbf{1}\left(p_{j} \leq p_{i}\right)\right]\right)^{k-1}\right] \simeq(v-r) \mu_{1},
$$


which can be transformed into a number $Q \geq K$ of population quantile restrictions:

$$
\sum_{j=1}^{n} \pi_{j}\left[\mathbf{1}\left(p_{j} \leq r+\frac{(v-r) \mu_{1}}{\sum_{k=1}^{K} k \mu_{k}\left(1-s_{\ell}\right)^{k-1}}\right)-s_{\ell}\right] \simeq 0
$$

for $s_{\ell} \in[0,1], \ell=1,2, \ldots, Q$. Using the lower bound defined in equation (5) one can eliminate marginal cost $r$ from these constraints. Then, using MEL based on these constraints, one can obtain estimates of the parameters $\left\{\mu_{k}\right\}_{k=1}^{K}$. Finally, by combining these estimates with the cutoff points in equation(2) obtained directly from the empirical CDF of prices, one gets $K$ points $\left\{\left(c_{k}, G\left(c_{k}\right)\right)\right\}_{k=1}^{K}$ of the search cost distribution. These points serve to construct an estimate of the search cost CDF.

Moraga-González and Wildenbeest (2008) put forward an alternative maximum likelihood (ML) method. There are two differences with respect to Hong and Shum's method. First, they compute the likelihood of a price as a function of the distribution of prices and exploit the equilibrium constancy-of-profits condition (4) to numerically calculate the value of the price CDF. In this way they obtain ML estimates of the parameters $\left\{\mu_{k}\right\}_{k=1}^{K}$. The second difference is that they introduce a method to compute ML estimates of the cutoffs by rewriting equation (2) as

$$
c_{k}=\int_{0}^{1} p(z)[(k+1) z-1](1-z)^{k-1} d z, k=1,2, \ldots, K-1 .
$$

where $p(z)$ is the inverse of the price distribution obtained from equation (4):

$$
p(z)=\frac{\mu_{1}(v-r)}{\sum_{k=1}^{K} k \mu_{k}(1-z)^{k-1}}+r .
$$

These two methods yield estimates of the points $\left\{\left(c_{k}, G\left(c_{k}\right)\right)\right\}_{k=1}^{K}$ of the search cost distribution. Under the standard regularity conditions, these points are estimated consistently. These two papers base their asymptotics on the number of prices $n$ going to infinity. Although one of the regularity conditions is identification of the points $\left\{\left(c_{k}, G\left(c_{k}\right)\right)\right\}_{k=1}^{K}$ of the search cost CDF, none of the earlier papers studied the identification issue. In the next section, we show that the sequence of points $\left\{\left(c_{k}, G\left(c_{k}\right)\right)\right\}_{k=1}^{K}$ is identified irrespective of whether $K$ is finite or infinite.

\subsection{Identification of the search cost cutoffs}

This subsection studies the conditions under which the econometrician can recover the unknowns of interest using price data. We assume that our data set has $n$ prices and our asymptotics is based on $n \rightarrow \infty$ (so the price distribution is known by the researcher). As before, assume $K$ denotes the maximum number of prices a consumer may search for in this market. ${ }^{6}$ We note that

\footnotetext{
${ }^{6}$ Our method of estimation, in contrast to the existing papers so far, allows for the case $K=n$, i.e., where consumers may observe all the prices in the market.
} 
the model transforms the distribution of prices in a market into a (countable) sequence of points $\left\{\left(c_{k}, G\left(c_{k}\right)\right)\right\}_{k=1}^{K}$ of the search cost CDF. In what follows, we maintain the assumption that this sequence exists for any $K$ (finite or infinite). ${ }^{7}$

The proposition below, proved in the Appendix, shows that if we know the price distribution $F$ and firms' cost $r$ is identified then we can identify the value of the search cost CDF corresponding to the cutoffs $\left\{c_{k}\right\}_{k=1}^{K}$, for $K$ finite or infinite.

Proposition 1 Suppose that the triplets of variables $\left(F,\left\{\mu_{k}\right\}_{k=1}^{K},\left\{c_{k}\right\}_{k=1}^{K}\right)$ and $\left(F^{\prime},\left\{\mu_{k}^{\prime}\right\}_{k=1}^{K},\left\{c_{k}^{\prime}\right\}_{k=1}^{K}\right)$ are generated by the triplets of variables $(G, v, r)$ and $\left(G^{\prime}, v^{\prime}, r^{\prime}\right)$, respectively, where $G$ and $G^{\prime}$ are distribution functions with support $(0, \infty)$ and positive density on this support. Suppose also that $F$ is a distribution function with support $(\underline{p}, v)$ and that $F^{\prime}=F$. In addition, assume that $r^{\prime}=r$. Then $\mu_{k}^{\prime}=\mu_{k}, c_{k}^{\prime}=c_{k}$ and $G^{\prime}\left(c_{k}\right)=G\left(c_{k}\right)$ for any $k \in\{1,2, \ldots, K\}$, that is, irrespective of whether $K$ is finite or infinite, the points of the search cost distribution corresponding to the sequence $\left\{c_{k}\right\}_{k=1}^{K}$ are identified.

As a consequence, this result establishes that the methods proposed earlier by Hong and Shum (2006) and Moraga-González and Wildenbeest (2008) identify the sequence of points $\left\{\left(c_{k}, G\left(c_{k}\right)\right)\right\}_{k=1}^{K}$ irrespective of whether $K$ is finite or infinite. Obviously, when $K$ is small, the sequence of points $\left\{\left(c_{k}, G\left(c_{k}\right)\right)\right\}_{k=1}^{K}$ may be insufficient to obtain a precise estimate of the search cost distribution in its full support. However, as shown in Figure 1, even if $K$ is relatively large the search cost cutoffs do not give much information on the magnitude of search costs at high quantiles. In this figure we plot the critical cutoff points $c_{k}$ for a different maximum number of prices a consumer may search $(K=10,15,50$, and 100). In these plots we set $v=500$ and $r=50$, and assume consumer search costs follow a log-normal distribution with parameters $(\nu, \sigma)=(0.5,5)$.

To overcome this problem, we propose to pool price data from various markets with similar search technology but different valuations or selling costs. Since the markets need to be similar in search costs, one can naturally take markets for different products that are purchased in a similar fashion. In this way, one could reasonably expect consumers to search for prices in the same manner and therefore incur similar costs. For example, one could take markets for different books, CDs, or DVD movies.

The effect of using data from multiple markets can be seen in Figure 2, where we plot the critical cutoff points $c_{k}$ obtained from using data from $M=1,5,25$, and 50 markets, for each of

\footnotetext{
${ }^{7}$ In connection with the first assumption, in Moraga-González, Sándor, and Wildenbeest (2010) we show an equilibrium always exists for arbitrary but finite $K$.
} 


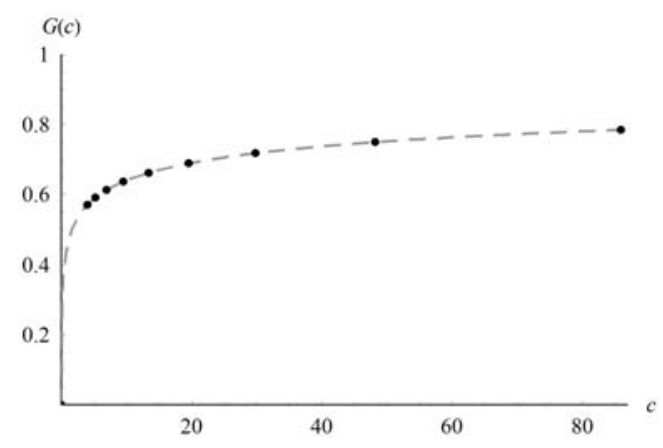

(a) $K=10$

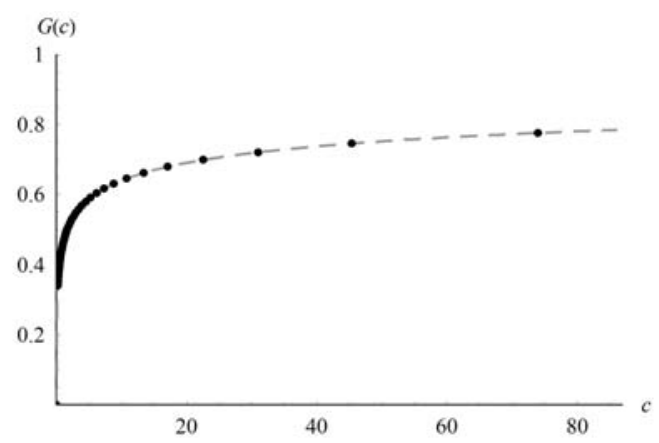

(c) $K=50$

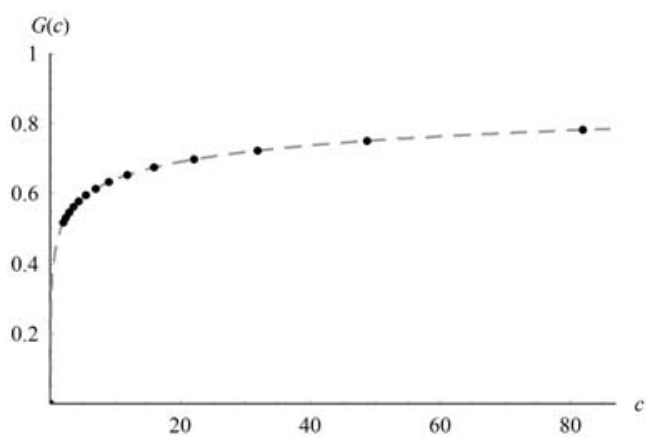

(b) $K=15$

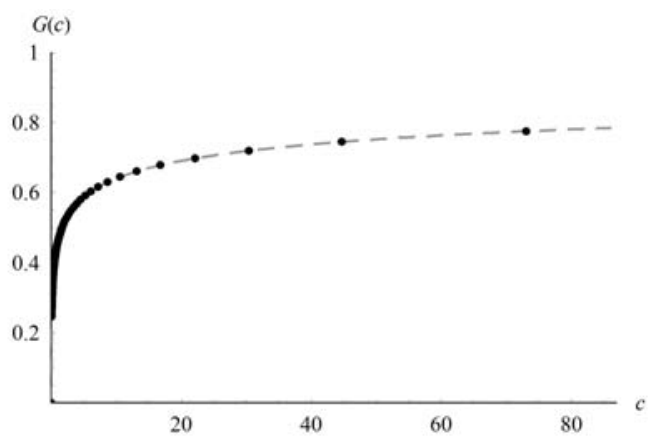

(d) $K=100$

Figure 1: Search cost cutoffs with data from only one market

the markets assuming the maximum number of prices a consumer can observe is 10 . In these plots we set $r=50$ and again assume consumer search costs follow a log-normal distribution with parameters $(\nu, \sigma)=(0.5,5)$. For the case of data from one market only we set $v^{m}=500$. For the situation with $M$ markets we take valuations in market $m$ as follows: $v^{m}=100+(500-100)(m-1) / M$, so the lowest consumer valuation is always 100 and if there are for example five markets we get $\left\{v^{m}\right\}_{m=1}^{5}=\{100,200,300,400,500\}$. The graphs make it clear that by using data from multiple markets we obtain much more information on the magnitude of search costs at high quantiles.

\subsection{Estimation}

As mentioned above, the previous studies on estimation of search cost distributions estimate first the parameters of the price distribution and then the search cost cutoff points. After this, they construct spline approximations of the search cost distribution by linking the estimated points by interpolation. In our framework we need an estimation method that exploits the link between the prices not only within a market but also across markets with the same underlying search cost distribution. For this purpose, we employ semi-nonparametric (SNP) maximum likelihood 


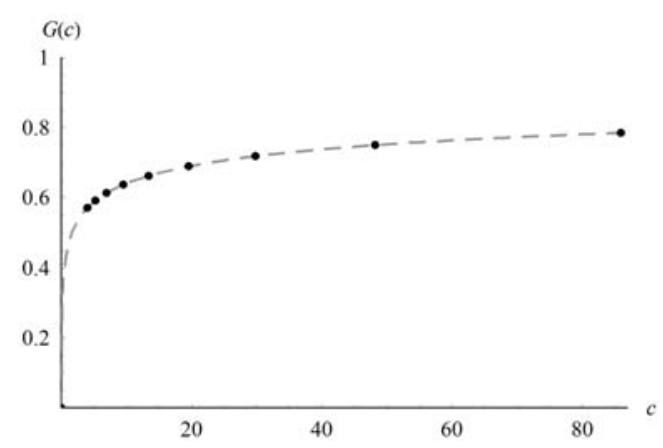

(a) $M=1, K=10$

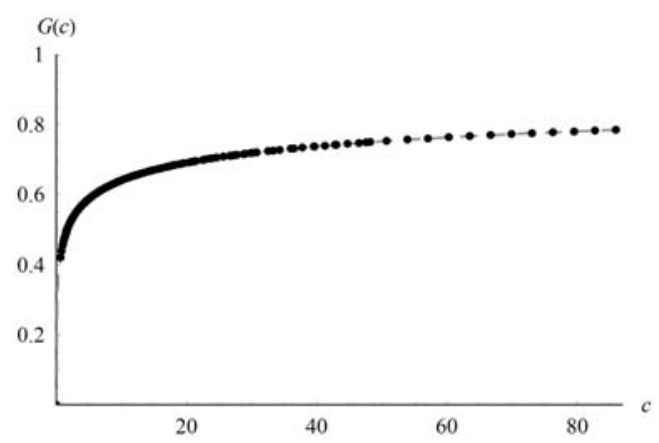

(c) $M=25, K=10$

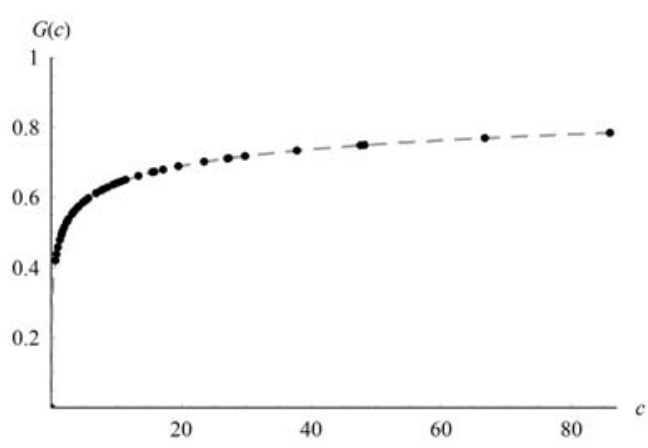

(b) $M=5, K=10$

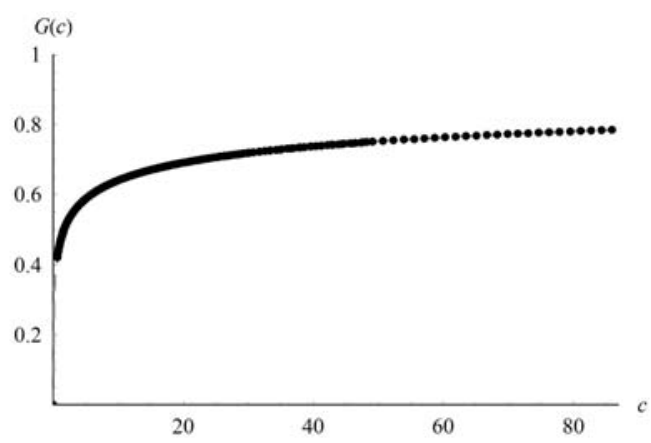

(d) $M=50, K=10$

Figure 2: Search cost cutoffs with data from $M$ different markets

estimation (Gallant and Nychka, 1987) and use the prices from all the markets at a time in our estimation procedure. This method is different because it takes directly the search cost distribution, which is common across markets, to be the parameter of the likelihood. In this sense, it exploits the data more efficiently than previous spline methods since those rely on estimating the parameters of the price distribution in every market separately and, therefore, ignore the link between the different data sets.

The idea behind SNP estimation is to use a flexible functional approximation of the search cost density. This functional approximation depends on a finite set of parameters to be estimated and this set can be made arbitrarily large as the number of observations goes to infinity. We construct our estimator of the search cost density by employing a flexible polynomial-type approximation, following the SNP estimation technique developed by Gallant and Nychka (1987).

The likelihood function can be constructed by deriving the density of prices in each market $m$ as a function of the search cost density $g$. Let $f^{m}(p \mid g)$ denote the density of price $p$ observed in a market $m$ given the search cost distribution $g$. Since the prices in a market $m$ are independent draws from $f^{m}(p \mid g)$, the log-likelihood function in market $m$ is $L L^{m}\left(g \mid \mathbf{p}_{m}\right)=\sum_{i=1}^{n_{m}} \log f^{m}\left(p_{i} \mid g\right)$ 
where $\mathbf{p}_{m}$ is the $n_{m}$-dimensional vector of prices in market $m=1,2, \ldots, M$. In order to compute this, first we apply the implicit function theorem to equation (4), which yields:

$$
f^{m}(p \mid g)=\frac{\sum_{k=1}^{K_{m}} k \mu_{k}^{m}\left(1-F^{m}(p \mid g)\right)^{k-1}}{\left(p-r^{m}\right) \sum_{k=1}^{K_{m}} k(k-1) \mu_{k}^{m}\left(1-F^{m}(p \mid g)\right)^{k-2}} .
$$

The quantities $\mu_{k}^{m}$ and $r^{m}$ in this expression need to be computed in terms of $g$. By solving equation (5) for $r^{m}$ we obtain an expression for the marginal cost in market $m$

$$
r^{m}=\frac{\underline{p}^{m} \sum_{k=1}^{K_{m}} k \mu_{k}^{m}-\mu_{1}^{m} v^{m}}{\sum_{k=2}^{K_{m}} k \mu_{k}^{m}} .
$$

We can estimate market $m$ 's lower and upper bounds of the price distribution $\underline{p}^{m}$ and $v^{m}$ (superconsistently) by taking the minimum and maximum price observed in the data, respectively. ${ }^{8}$ Then, for every market $m$, we compute $\left\{c_{k}^{m}\right\}_{k=1}^{K_{m}}$ from equations (8) in terms of $g,{ }^{9}$ and then use equations $(3 c),(3 d),(4)$, and (10) to find the values of $F^{m}\left(p_{i} \mid g\right)$ and $f^{m}\left(p_{i} \mid g\right)$. In this way we obtain the joint log-likelihood of all markets as a function of $g: L L\left(g \mid \mathbf{p}_{\mathbf{1}}, \mathbf{p}_{\mathbf{2}}, \ldots, \mathbf{p}_{\mathbf{M}}\right)=\sum_{m=1}^{M}\left(\sum_{i=1}^{n_{m}} \log f^{m}\left(p_{i} \mid g\right)\right)$.

For the polynomial-type parametric function that estimates the search cost density we employ the SNP density estimator of Gallant and Nychka (1987). This SNP estimator is based upon a Hermite polynomial expansion. The idea behind their SNP procedure is that any reasonable density can be mimicked by such a Hermite polynomial series. SNP density estimators are essentially nonparametric because the set of all Hermite polynomial expansions is dense in the set of density functions that are relevant (Gallant and Nychka, 1987). ${ }^{10}$

To apply the SNP estimation in our problem, we specify the search cost density as follows:

$$
g(c ; \gamma, \sigma, \theta)=\frac{\left[\sum_{i=0}^{N} \theta_{i} u_{i}(c)\right]^{2}}{\sum_{i=0}^{N} \theta_{i}^{2}}, \theta \in \Theta_{N}
$$

where $\Theta_{N}=\left\{\theta: \theta=\left(\theta_{0}, \theta_{1}, \ldots, \theta_{N}\right), \theta_{0}=1\right\}, N$ is the number of polynomial terms and

$$
\begin{aligned}
& u_{0}(c)=(c \sigma \sqrt{2 \pi})^{-1 / 2} \mathrm{e}^{-((\log c-\gamma) / \sigma)^{2} / 4} \\
& u_{1}(c)=(c \sigma \sqrt{2 \pi})^{-1 / 2}((\log c-\gamma) / \sigma) \mathrm{e}^{-((\log c-\gamma) / \sigma)^{2} / 4} \\
& u_{i}(c)=\left[((\log c-\gamma) / \sigma) u_{i-1}(c)-\sqrt{i-1} u_{i-2}(c)\right] / \sqrt{i}, \text { for } i \geq 2
\end{aligned}
$$

\footnotetext{
${ }^{8}$ In a similar fashion, order statistics are also used to estimate the lower and upper bound of distributions of bids (see e.g. Donald and Paarsch, 1993).

${ }^{9}$ In markets with many cutoff points solving this nonlinear system of equations may be time consuming. One alternative is to estimate the cutoffs directly by the empirical price CDF. The trade off is precision of the estimates against computational time.

${ }^{10} \mathrm{SNP}$ has recently been applied to the estimation of labor search frictions (Koning, van den Berg, and Ridder, 2000), labor supply (Van Soest, Das, and Gong, 2002), travel demand (Van der Klaauw and Koning, 2003), and auctions (Brendstrup and Paarsch, 2006).
} 
This parametric form corresponds to the univariate SNP estimator studied extensively by Fenton and Gallant (1996). Our expressions are obtained by transforming their random variable $x$ with the density defined in their Section 4.3 into $c=\exp ^{\gamma+\sigma x}$. This transformation is useful in our case since search costs are positive. The vector of parameters to be estimated by maximum likelihood is $\left\{\gamma, \sigma, \theta_{1}, \ldots, \theta_{N}\right\}$ and $N$ can be made arbitrarily large as the number of observations increases to infinity.

Gallant and Nychka (1987) provide conditions on the unknown density (e.g. differentiability and restricted tail behavior) under which their estimator is consistent using i.i.d. observations. Since our estimator of the search cost density uses data from multiple markets, Hoadley's (1971) conditions for the consistency of maximum likelihood estimators with independently non-identically distributed observations also need to be satisfied.

\subsection{Monte Carlo Study}

To study the small sample properties of the estimator we set up a Monte Carlo study. We focus on the estimation of the two different search cost distributions, in particular

$$
g(c)=0.5 \cdot \operatorname{lognormal}(c, 2,10)+0.5 \cdot \operatorname{lognormal}(c, 3,0.2)
$$

and

$$
g(c)=0.25 \cdot \operatorname{gamma}(c, 1,1)+0.5 \cdot \operatorname{gamma}(c, 30,1)+0.25 \cdot \operatorname{gamma}(c, 100,1) .
$$

where lognormal $(c, a, b)$ and gamma $(c, a, b)$ refer to the densities of the lognormal and gamma distributions with parameters $a$ and $b$, respectively. The setup of the study is as follows. We take $M=20$ markets. Each market has the same search cost distribution $G(c)$ but a different valuation net of marginal cost, $v^{m}-r^{m}$. The 20 values for $v^{m}-r^{m}$ are drawn from a lognormal distribution with parameters 5 and 1 . For each market $m$, we set the maximum number of prices a consumer can observe $K_{m}$ equal to 10 . With the parameters of a market $m$ at hand, we compute the market equilibrium by numerically solving the system of equations (8). Given the cutoff values for a market $m$, we construct the equilibrium price distribution in that market $m$ using equation (9). Next, we randomly draw 50 prices from each equilibrium price distribution $F_{m}$ and use all 1,000 prices as an input for the SNP estimation procedure. The estimation is repeated 100 times. ${ }^{11}$ For the number

\footnotetext{
${ }^{11}$ To gain computing time we use the empirical distribution of prices in each market to estimate the $c_{k}$ 's. This is likely to lead to less precise estimates, so our results should be seen as a lower bound on the performance of the estimator when using equation (8) instead.
} 
of polynomial terms $N$ in equation (12) we set $N=5 .{ }^{12}$

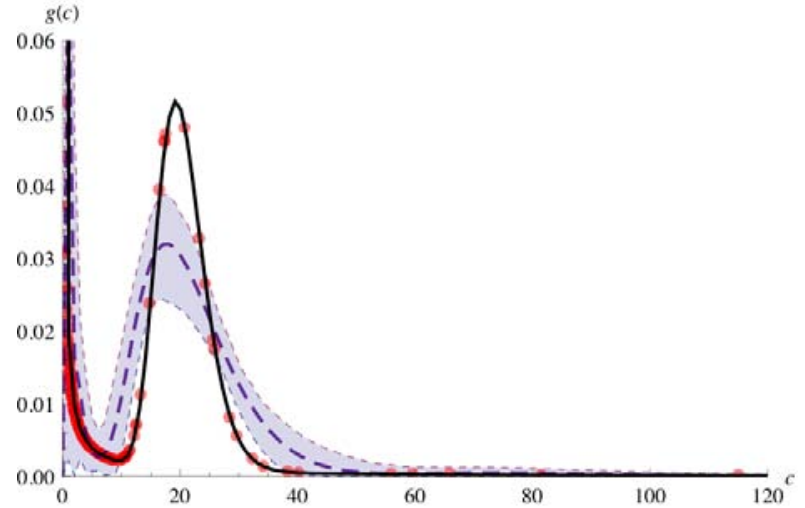

(a) Mixture lognormals: equation (13)

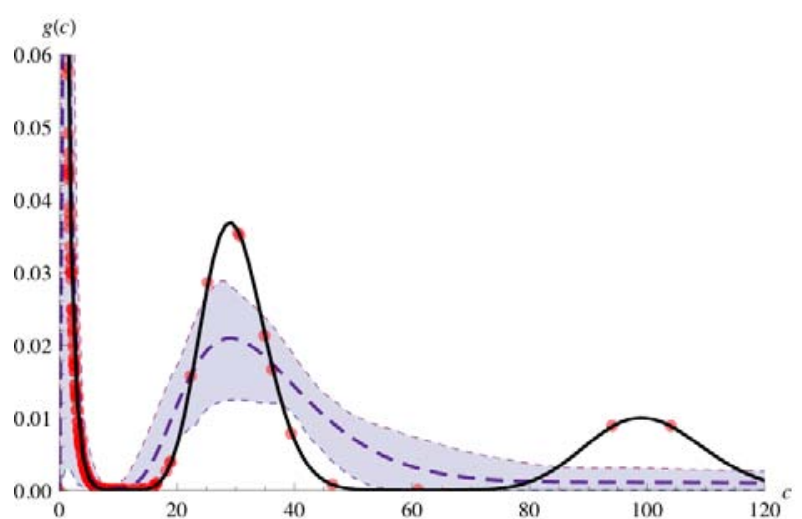

(b) Mixture gammas: equation (14)

Figure 3: Monte Carlo results: estimated search cost PDF

Figure 3 shows the results of the study. For each true search cost distribution, we report $(i)$ the corresponding mean and the $90 \%$ confidence interval of its 100 estimates, and $(i i)$ the search cost cutoffs that we can estimate given that data. Figure 3(a) corresponds to the case where the search cost density is a mixture of two lognormal densities (equation (13)), while Figure 3(b) corresponds to the mixture of three gamma densities (equation (14)). In both graphs, the solid curve represents the true search cost PDF, while the thick dashed curve shows the mean of the 100 estimations. The $90 \%$ confidence interval is given by the shaded area between the thin dashed curves. The search cost cutoffs are depicted by the red dots.

In spite of the relatively small number of markets and observations per market, the figures illustrate that our estimation procedure performs fairly well. The estimates mimic the true shape of the search cost densities relatively well at the lower quantiles. The small third "bump" of the density in Figure 3(b) is not captured that well by the average estimate. This can be explained by the low number of search cost cutoffs (red dots) in the neighborhood of 100 . If we were to add more markets with relatively high valuation to our data set, the number of search cost cutoffs in the neighborhood of 100 would increase and this would improve the outcome of the estimation.

\footnotetext{
${ }^{12}$ According to Fenton and Gallant(1996), the number of polynomial terms that is asymptotically optimal is given by $\left(M \times n_{m}\right)^{1 / 5}$, which in our case is approximately equal to 4 . We have experimented with $N=3, N=4$ and $N=5$ and chosen to report the results for the case $N=5$.
} 


\section{Application}

In this section we use the SNP estimation method described above to quantify search costs in realworld markets for memory chips. We focus on computer memory chips for notebooks (so called SO-DIMM, or Small Outline Dual In-line Memory Module). Since we need products from different markets, we select memory chips produced for different brands and types of notebooks. Table 1 gives the details of the 10 products we include in our data set. There are several reasons for choosing these memory chips. First, since all the chips are sold online, we expect search costs to be similar across markets. Second, even though all memory chips are manufactured by Kingstonthe largest producer in the sector - each memory chip in our sample is meant to be used in a particular notebook brand only_including Toshiba, Dell, Acer, IBM and HP Compaq. Given that substitutability across products is somewhat limited due to technical reasons, we shall assume that different microchips belong in separate markets so the use of a search model with homogeneous products is reasonable. All the memory chips were somewhat at the top of the product line at the time of data collection. In particular they exhibit relatively large storage capacity (1 gigabyte) and fast speed of operation (most of them above $400 \mathrm{MHz}$ ). Given the large storage capacity of the memory chips in the data set, most consumers would only consider to buy one memory chip, so the single-unit inelastic demand assumption of the theoretical model seems also reasonable.

\begin{tabular}{llllll}
\hline \hline Part number & Manufacturer & Compatibility & Size & Speed & Form factor \\
\hline KTT3311A & Kingston & Toshiba & 1GB & 333MHz DDR333/PC2700 & 200-pin SoDIMM \\
KTT533D2 & Kingston & Toshiba & 1GB & 533MHz DDR2-533/PC2-4200 & 200-pin SoDIMM \\
KTD-INSP8200 & Kingston & Dell & 1GB & 266MHz DDR266/PC2100 & 200-pin SoDIMM \\
KTD-INSP5150 & Kingston & Dell & 1GB & 333MHz DDR333/PC2700 & 200-pin SoDIMM \\
KTD-INSP6000 & Kingston & Dell & 1GB & 533MHz DDR2-533/PC2-4200 & 240-pin SoDIMM \\
KTD-INSP6000A & Kingston & Dell & 1GB & 533MHz DDR2-533/PC2-4200 & 200-pin SoDIMM \\
KAC-MEME & Kingston & Acer & 1GB & 533MHz DDR2-533/PC2-4200 & 200-pin SoDIMM \\
KTD-INSP9100 & Kingston & Dell & 1GB & 400MHz DDR400/PC3200 & 200-pin SoDIMM \\
KTM-TP3840 & Kingston & IBM & 1GB & 533MHz DDR2-533/PC2-4200 & 200-pin SoDIMM \\
KTH-ZD8000A & Kingston & HP Compaq & 1GB & 533MHz DDR2-533/PC2-4200 & 200-pin SoDIMM \\
\hline \hline
\end{tabular}

Table 1: List of products

For all the memory chips in the data set we collected online prices charged in the United States, in February 2006. To obtain a sufficiently representative sample, we gathered product and price information from several sources at the same time. We proceeded as follows. We first visited the price comparison sites shopper.com and pricegrabber.com and collected the names of all the shops that were seen active in markets for memory chips; in total we found 49 stores. If for a particular product we saw a shop quoting its price on shopper.com and/or pricegrabber.com, we took the 
price directly from the price comparison site; otherwise we visited the web-address of the vendor to check if the product was available and at what price it was offered. Table 2 gives some summary statistics of the data set. The number of firms quoting prices in each market is relatively large, ranging from 24 to 41 . In our study we estimate the maximum number of prices consumers observe in each market $K_{m}$ by the number of firms that were observed to be quoting prices in that market.

\begin{tabular}{lccccc}
\hline \hline Part number & No. of Stores & Mean Price (Std) & Min. Price & Max. Price & Coeff. of Var. (as \%) \\
\hline KTT3311A & 32 & $181.67(24.62)$ & 148.62 & 235.00 & 13.55 \\
KTT533D2 & 33 & $123.33(15.62)$ & 100.45 & 161.40 & 12.66 \\
KTD-INSP8200 & 39 & $173.59(21.31)$ & 148.62 & 249.54 & 12.28 \\
KTD-INSP5150 & 39 & $179.09(19.84)$ & 148.62 & 222.35 & 11.08 \\
KTD-INSP6000 & 35 & $120.29(13.48)$ & 100.45 & 151.05 & 11.21 \\
KTD-INSP6000A & 38 & $116.33(13.43)$ & 94.99 & 154.50 & 11.54 \\
KAC-MEME & 24 & $123.58(17.47)$ & 101.92 & 161.64 & 14.14 \\
KTD-INSP9100 & 33 & $175.84(24.38)$ & 148.62 & 249.54 & 13.87 \\
KTM-TP3840 & 37 & $122.83(14.32)$ & 104.55 & 161.94 & 11.65 \\
KTH-ZD8000A & 41 & $116.77(12.25)$ & 100.45 & 154.50 & 10.49 \\
\hline \hline
\end{tabular}

Table 2: Summary statistics

Our model assumes consumers search non-sequentially. Clearly, in an electronic environment the reality is more complex than this. Many consumers (bargain hunters) typically proceed by first visiting a shopbot (for instance shopper.com or pricegrabber.com) followed by sequentially visiting the firms listed in the shopbot. This procedure resembles the optimal search protocol described in Morgan and Manning (1985), which is hybrid in nature. In addition, it has been pointed out (see for example Whelan, 2001) that some consumers are somewhat loyal (convenience shoppers). Given these characteristics, our assumed search strategy should then be seen as an approximation to a more realistic search behavior and, as a result, the results of this section should be interpreted cautiously.

Almost all memory chips are priced above 100 US dollars. For all products we observe significant price dispersion as measured by the price range (difference between the maximum and the minimum prices) and by the coefficient of variation. The benefits to a consumer from searching are significant; in particular, the gains from being fully informed relative to buying from a shop at random in these markets range from 16.32 to 33.05 US dollars. As mentioned above, we estimate the valuation of a memory chip by the maximum price observed in the market.

The prices used for our estimations include neither shipping costs nor sales taxes. The main reason for this omission is that shipping costs and sales taxes depend on the state in which the consumer resides, which makes it difficult to compare total prices. However, for robustness purposes, 
we estimated the model neglecting sales taxes but using the shipping costs as if we were living in New York. The qualitative nature of the results did not change.

Although the memory chips themselves are completely homogeneous, the price differences across vendors for a given chip may be due to store differentiation. Consumers might prefer one shop over another on the basis of observable store characteristics like quality ratings, return policies, stock availability, order fulfillment, payment methods, etc. To see the impact of observable shop characteristics on prices, we regressed prices on indicators that are readily available from the price comparison sites. More precisely, we estimated the following model:

$$
P R I C E_{j}=\beta_{0}+\beta_{1} \cdot R A T I N G_{j}+\beta_{2} \cdot D I S C L O S E_{j}+\beta_{3} \cdot S T O C K_{j}+\beta_{4} \cdot L O G O_{j}+\varepsilon_{j}
$$

where, for each product, $P R I C E_{j}$ is the list price of store $j, R A T I N G_{j}$ is an average of the ranking of store $j$ on shopper.com and pricegrabber.com, DISCLOSE $E_{j}$ is a dummy for whether shop $j$ disclosed shipping cost on either shopper.com or pricegrabber.com, $S T O C K_{j}$ is a dummy for whether shop $j$ had the item in stock, and $L O G O_{j}$ is a dummy for whether shop $j$ had its logo on either shopper.com or pricegrabber.com. We estimated this equation by OLS. The resulting $R$-squared values indicate that only between $3 \%$ and $27 \%$ of the total variation in prices can be attributed to observable differences in store characteristics. ${ }^{13}$ Although this does not rule out that there are unobservable differences between stores (e.g., cost differences or branding), this does suggest that the observable characteristics cannot explain the vast majority of variation in prices and that something else must cause such variability. In spite of this, for robustness purposes, we also estimated the model using the residuals of the regression above. This is standard practice in many structural auction models (e.g., Haile, Hong, and Shum, 2003; Bajari, Houghton, and Tadelis, 2007; An, Hu, and Shum, 2010). As shown by Haile, Hong, and Shum (2003) for first-price sealed bid auctions, this approach is justified as long as prices are additively separable in a common storespecific component related to the observed store characteristics and in an idiosyncratic component. The estimates of the model using residuals are similar to the estimates using prices (see footnote 15 and Figure 4 below).

Because we only observe the stores' prices at one moment in time, we cannot check whether stores indeed randomize their prices over time, as predicted by our search model. However, using a different data set Moraga-González and Wildenbeest (2008) show that firms indeed seem to randomize in the online market for memory chips; at the same time, other studies find evidence for

\footnotetext{
${ }^{13}$ For all memory chips, all the OLS coefficient estimates were not significant except the coefficient for $L O G O_{j}$, which was positive and significant at a 5\% level for the KTM-TP3840 and KTH-ZD8000A chips.
} 


\begin{tabular}{lc}
\hline \hline & SNP \\
\hline$p^{n}$ & 3 \\
$\#$ obs & 351 \\
$\gamma$ & $0.941(0.125)$ \\
$\sigma$ & $0.923(0.040)$ \\
$\theta_{0}$ & 1.000 \\
$\theta_{1}$ & $0.332(0.181)$ \\
$\theta_{2}$ & $-0.296(0.158)$ \\
$\theta_{3}$ & $-1.310(0.235)$ \\
LL & 1309.87 \\
\hline \hline \multicolumn{2}{l}{ Notes: Estimated standard errors } \\
in parenthesis.
\end{tabular}

Table 3: Parameter estimates SNP function

mixed strategies in other markets (e.g., Lach (2002) for chicken, refrigerators, coffee, and flour in Israel; and Wildenbeest (2009) for grocery products in the United Kingdom).

Table 3 presents the SNP estimation results. We follow the procedure explained in Section 3.3 and the recommendation by Fenton and Gallant (1996) and set $N=3$, which equals the closest integer to the fifth root of the total number of observations. ${ }^{14}$ Table 3 shows that all parameter estimates are significant at a $1 \%$ level, except for $\theta_{1}$ and $\theta_{2}$, which are significant at a $5 \%$ level. ${ }^{15}$ The standard errors reported in the table are meaningful in the case when the presented model is the true parametric model. The solid curves in Figures 4(a) and 4(b) denote the estimated search cost CDF and PDF respectively. These graphs also show how the estimated search cost cutoffs (red dots) cover the support of the search cost distribution. The dashed curves are the estimated search cost CDF and PDF if we use the residuals of the regression model specified in equation (15). The graph illustrates that both are very similar.

Using the estimates of the parameters of the SNP specification in Table 3 we can compute the mean, the median, and the standard deviation of the unobserved search cost distribution. The median consumer has a search cost equal to 5.05 US dollars. On average a consumer has a search cost value equal to 13.41 US dollars and the standard deviation is 24.49 US dollars. It is also interesting to investigate the distribution of search intensities in these markets. Since each market has specific parameters, even though search costs are assumed to be similar, it is unlikely that consumer behavior will be the same across markets. Table 4 shows that it is indeed the case that search intensities are different across markets. For example, $18 \%$ of consumers search once for

\footnotetext{
${ }^{14}$ In cases when there are sufficiently many observations, as is the case in our data set, we can use the empirical distribution of prices in each market directly to estimate the $c_{k}$ 's. The gain in computing time is huge and the results for our data are very similar.

${ }^{15}$ When we use the residuals of the regression model specified in equation (15) to estimate the model, the estimates obtained are the following: $\gamma=0.697, \sigma=0.778, \theta_{0}=1.000, \theta_{1}=0.264, \theta_{2}=-0.382$, and $\theta_{3}=-1.668$.
} 


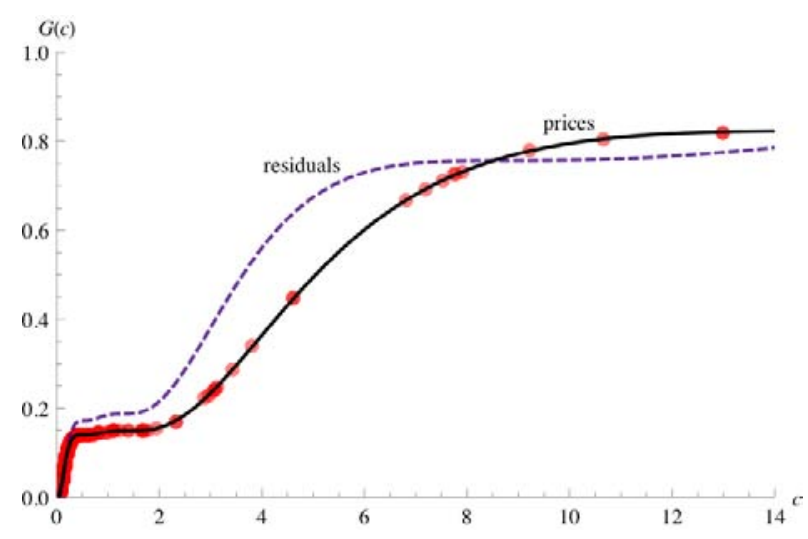

(a) Search cost CDF

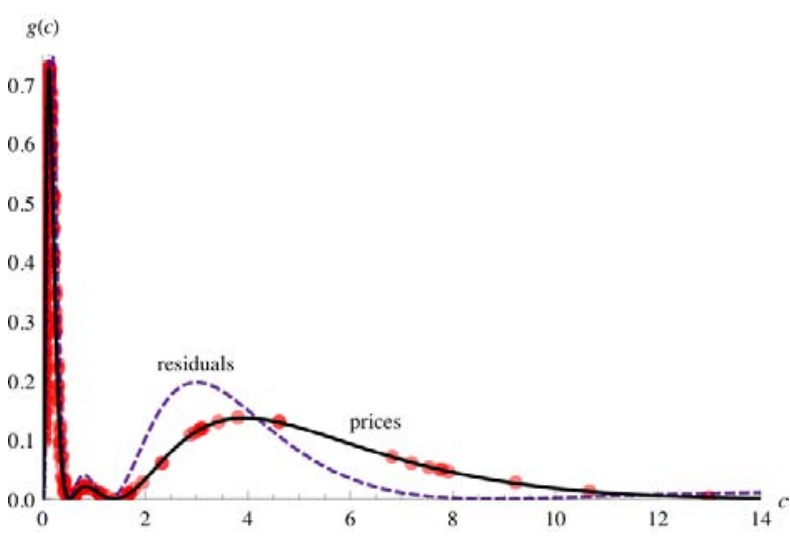

(b) Search cost PDF

Figure 4: Estimated search cost distribution

the KTD-INSP9100 memory chip, while 33\% searches once for the KTD-INSP6000 memory chip. Similarly, for the KTD-INSP9100 chip 37\% of consumers searches twice, while $49 \%$ searches twice for the KTD-INSP5150G memory chip. However, the share of consumers searching at most three times is more or less similar across markets; approximately $85 \%$ of the consumers have search cost above $\$ 2$ and search for at most three prices. Table 4 also illustrates that the group of consumers searching for between 4 and 10 firms is with percentages between 2 and 4 relatively small. About $13 \%$ of consumers search with more than 10 times thoroughly, which means they have search costs less than 30 dollar cents. Figures 4(a) and 4(b) show that the consumers can roughly be divided into three groups: buyers who do not search, buyers who compare at most three prices and buyers who compare many prices in the market. In sum, we conclude that consumers have either quite high search costs or quite low search costs.

The gray dots in Figures 4(a) and 4(b) denote the cutoffs on the search cost CDF and PDF, respectively. Not surprisingly, given that we have data from only 10 markets, most cutoffs are found at low search cost values. As explained in Section 3.2, adding extra markets will increase the number of cutoffs for higher search cost values.

The fact that a significant proportion of consumers does not compare prices gives substantial market power to the firms. Using the estimates of the SNP specification, we can retrieve the marginal cost $r$ in each market, which is also reported in Table 4. Marginal costs range between $57 \%$ and $65 \%$ of the value of the product so the average price-cost margins range between $17 \%$ and $23 \%$ across markets. We calculate standard errors for $r$ using the delta method. All the estimated values for $r$ are highly significant. 


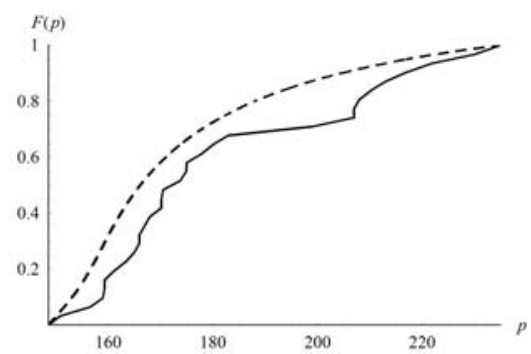

(a) KTT3311A

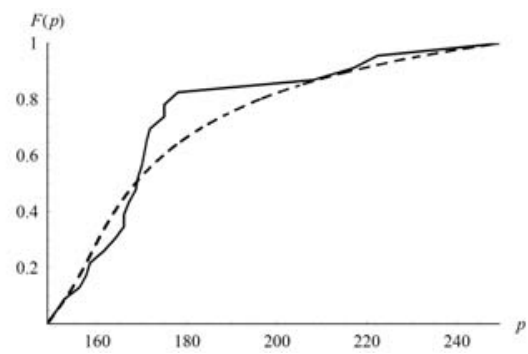

(c) KTD-INSP8200

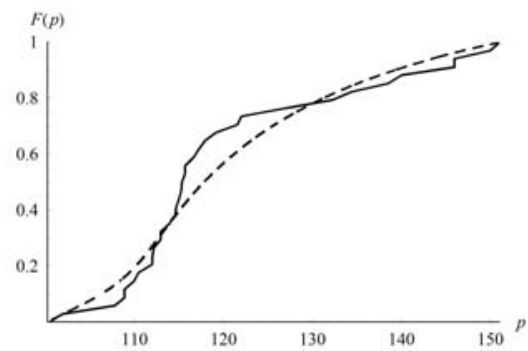

(e) KTD-INSP6000

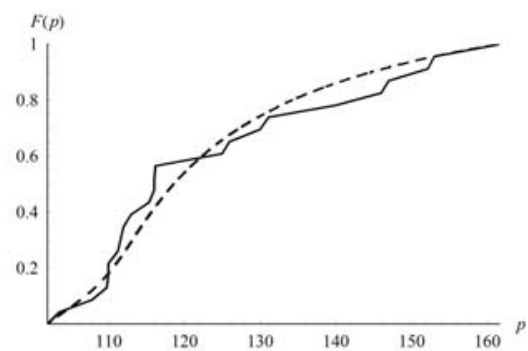

(g) KAC-MEME

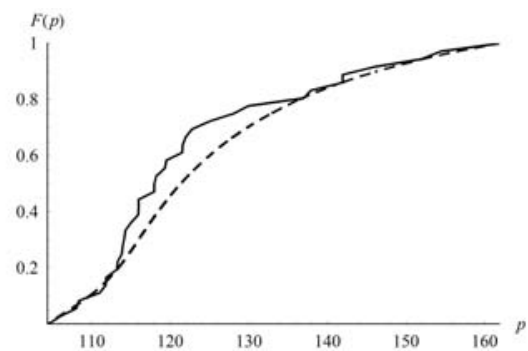

(i) KTM-TP3840

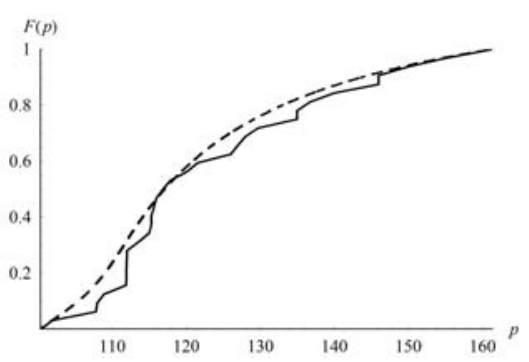

(b) KTT533D2/1G

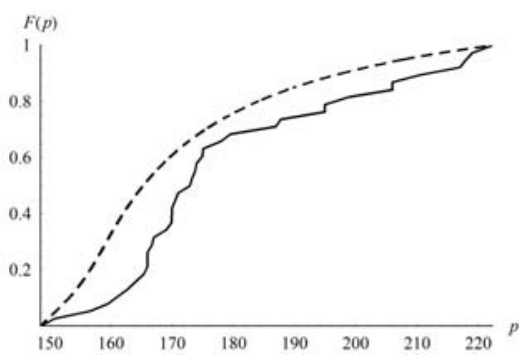

(d) KTD-INSP5150

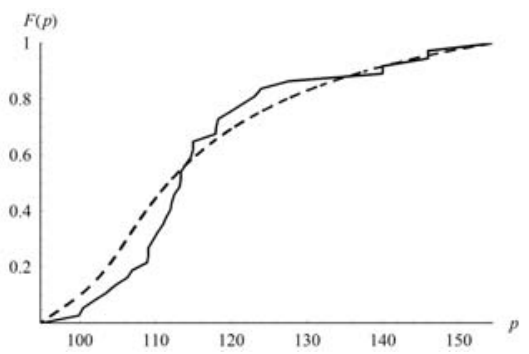

(f) KTD-INSP6000A

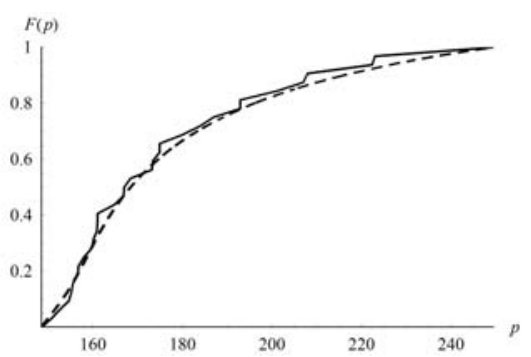

(h) KTD-INSP9100

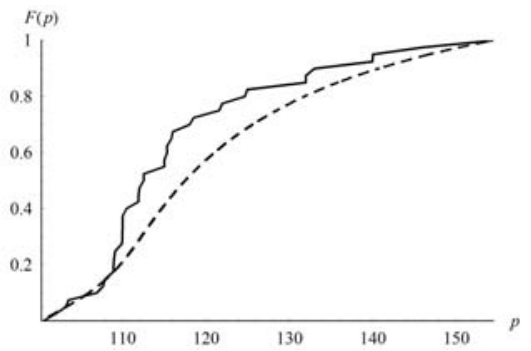

(j) KTH-ZD8000A

Figure 5: Estimated and empirical price CDF's 


\begin{tabular}{|c|c|c|c|c|c|c|c|c|c|c|c|c|c|}
\hline Part number & $\bar{~} \bar{K}$ & $p$ & $v$ & $r$ & $\mu_{1}$ & $\mu_{2}$ & $\mu_{3}$ & $\mu_{4}$ & $\mu_{5}$ & $\mu_{6 \ldots 10}$ & $\mu_{11 \ldots 15}$ & $\mu_{16 \ldots K}$ & $K S$ \\
\hline KTT3311A & 32 & 148.62 & 235.00 & $144.23(1.70)$ & 0.19 & 0.47 & 0.18 & 0.01 & 0.00 & 0.02 & 0.06 & 0.07 & 1.24 \\
\hline KTT533D2 & 33 & 100.45 & 161.40 & $95.86(1.89)$ & 0.27 & 0.48 & 0.10 & 0.00 & 0.01 & 0.01 & 0.05 & 0.07 & 0.91 \\
\hline KTD-INSP8200 & 39 & 148.62 & 249.54 & $144.08(1.64)$ & 0.18 & 0.37 & 0.28 & 0.02 & 0.00 & 0.02 & 0.06 & 0.07 & 1.20 \\
\hline KTD-INSP5150G & 39 & 148.62 & 222.35 & $144.13(1.77)$ & 0.22 & 0.49 & 0.13 & 0.00 & 0.01 & 0.02 & 0.06 & 0.07 & 2.01 \\
\hline KTD-INSP6000 & 35 & 100.45 & 151.05 & $95.67(2.02)$ & 0.33 & 0.44 & 0.07 & 0.00 & 0.00 & 0.01 & 0.05 & 0.09 & 0.92 \\
\hline KTD-INSP6000A & 38 & 94.99 & 154.50 & $90.39(1.90)$ & 0.28 & 0.48 & 0.09 & 0.00 & 0.01 & 0.01 & 0.05 & 0.07 & 1.20 \\
\hline KAC-MEME & 24 & 101.92 & 161.64 & $97.36(1.93)$ & 0.27 & 0.48 & 0.09 & 0.00 & 0.01 & 0.01 & 0.06 & 0.07 & 0.80 \\
\hline KTD-INSP9100 & 33 & 148.62 & 249.54 & $144.08(1.64)$ & 0.18 & 0.37 & 0.28 & 0.02 & 0.00 & 0.02 & 0.06 & 0.07 & 0.57 \\
\hline KTM-TP3840 & 37 & 104.55 & 161.94 & $99.91(1.93)$ & 0.29 & 0.47 & 0.09 & 0.00 & 0.01 & 0.01 & 0.05 & 0.08 & 0.98 \\
\hline KTH-ZD8000A & 41 & 100.45 & 154.50 & $95.75(1.97)$ & 0.31 & 0.46 & 0.08 & 0.00 & 0.00 & 0.01 & 0.05 & 0.08 & 1.49 \\
\hline
\end{tabular}

Table 4: Parameter estimates products and fit

To test whether the estimated model explains observed prices well, we calculate the KolmogorovSmirnov statistic (KS-test) in each individual market. The KS-test statistic is based on the maximum difference between the empirical price CDF and the estimated price CDF, which is the computed price equilibrium given the estimate of the search cost distribution. The null hypothesis for this test is that the distributions are similar, the alternative hypothesis is that the empirical and the estimated price CDF are different. Table 4 gives the KS-test results and since for the majority of products the $K S$ value is below the $95 \%$-critical value of the KS-statistic of 1.36 , for eight out of ten memory chips we cannot reject the null-hypothesis that the prices are drawn from the estimated price CDF. ${ }^{16}$ The goodness-of-fit is also shown in Figure 5, where we have plotted both the empirical and the estimated price CDF for each market. A solid curve represents the empirical price CDF, while a dashed curve represents an estimated price CDF; the graphs show that both curves are quite close to each other for most products.

\section{Conclusions}

Since the seminal contribution of Stigler (1961), economists have dedicated a significant amount of effort to understand the nature of competition in markets where price information is not readily available to consumers. One of the lessons learned is that consumer search models may lead to predictions different from those obtained from conventional economic theory. Another is that the particular direction of the effects of public policy measures such as the introduction of taxes or the dismantling of barriers to entry depends on the shape of the search cost distribution. These observations motivate the development of methods to estimate search costs.

\footnotetext{
${ }^{16}$ We have calculated $K S$ in Table 4 as $\sqrt{n_{m}} \cdot \tau_{n_{m}}$, where $n_{m}$ is the number of price observations for the specific memory chip and $\tau_{n_{m}}$ is the maximum absolute difference over all prices between the estimated price CDF and the empirical price CDF.
} 
This paper has argued that in order to increase the precision of the estimates one needs to increase the number of estimated critical search cost cutoffs at all quantiles of the search cost distribution, which can be done by pooling price data from various markets with similar search technology and different valuations. To take advantage of the relationship between markets we have proposed a new method to estimate the search cost density function by a semi-nonparametric density estimator whose parameters maximize the joint likelihood corresponding to all the markets. The paper has also illustrated the potential of our method by applying it to a data set of online prices for ten notebook memory chips. The estimates obtained suggest that the search cost density is essentially bimodal such that a large fraction of consumers searches very little and a small fraction of consumers samples a relatively large number of stores.

Along the way we have made several simplifying assumptions. One of the assumptions has been that consumers have the same valuation. In future work, we would like to relax this assumption and study a framework where there is consumer valuation and search cost heterogeneity. One of the advantages of developing such a framework is that it would enable the econometrician to estimate the correlation between consumer valuations and search costs. Another important assumption has been that firms are symmetric, i.e., they have the same marginal costs of production. Since marginal cost heterogeneity may be an important factor behind the observed price variation in realworld markets, future work should allow for firm heterogeneity. Such a framework would help the researcher separate price variation caused by search costs from that caused by cost heterogeneity. 


\section{APPENDIX}

Proof of Proposition 1. We present the proof in the case when $K=\infty$; the case of finite $K$ follows the same ideas and therefore is skipped. By assumption, $r^{\prime}=r$ and since $F=F^{\prime}$ we have $v^{\prime}=v$. First we show that $\mu_{k}^{\prime}=\mu_{k}$ for any $k$. For this we note first that neither $\mu_{1}$ nor $\mu_{1}^{\prime}$ can be equal to zero. If $\mu_{1}=0$ then by equation (4) $\sum_{k \geq 2} k \mu_{k}(1-F(p))^{k-1}=0$ for any $p \in(\underline{p}, v)$, which, due to the fact that $F$ is strictly increasing and continuous, can only happen if $\mu_{k}=0$ for any $k \geq 2$. This further implies by equations $(3 c)$ and $(3 d)$ that $G\left(c_{k}\right)=1$ for any $k \geq 1$. Because the support of $G$ is $(0, \infty)$, this can only happen if $c_{k}=\infty$ for any $k \geq 1$. By equation (2), $c_{k}=E p_{1: k}-E p_{1: k+1}$, where $E p_{1: k}=E\left[\min \left\{p_{1}, \ldots, p_{k}\right\}\right]$. Since $E p_{1: k} \geq E p_{1: k+1}$ and $E p_{1: k} \geq \underline{p}$ for any $k$, the series $\left(E p_{1: k}\right)_{k \geq 11}$ is convergent. Hence $c_{k} \rightarrow 0$ as $k \rightarrow \infty$, a contradiction with $c_{k}=\infty$ for any $k \geq 1$. Therefore, $\mu_{1}>0$. Since exactly the same arguments apply to $\mu_{1}^{\prime}$, we have shown that $\mu_{1}$ and $\mu_{1}^{\prime}$ are strictly positive.

From equation (4) we obtain

$$
\sum_{k \geq 1} k \frac{\mu_{k}}{\mu_{1}}(1-F(p))^{k-1}=\frac{v-r}{p-r}=\sum_{k \geq 1} k \frac{\mu_{k}^{\prime}}{\mu_{1}^{\prime}}(1-F(p))^{k-1} \quad \text { for any } p \in(\underline{p}, v] .
$$

This is equivalent to

$$
\sum_{k \geq 2} \lambda_{k} t^{k-1}=0 \quad \text { for any } t \in(0, \alpha),
$$

where $\lambda_{k}=k\left(\frac{\mu_{k}}{\mu_{1}}-\frac{\mu_{k}^{\prime}}{\mu_{1}^{\prime}}\right)$ for $k \geq 1$ and $t=1-F(p)$. This latter transformation is possible because $F$ is strictly increasing on some interval $(\widetilde{p}, v)$, where $1-F(\widetilde{p})=\alpha$. We now invoke Lemma A.1, which is presented below. This lemma implies that equation (A16) can only hold if $\lambda_{k}=0$ for any $k \geq 2$. Therefore $\frac{\mu_{k}}{\mu_{1}}=\frac{\mu_{k}^{\prime}}{\mu_{1}^{\prime}}$. On the other hand, $\mu_{1}+\sum_{k \geq 2} \mu_{k}=\mu_{1}^{\prime}+\sum_{k \geq 2} \mu_{k}^{\prime}=1$. Together these equalities imply $\frac{1}{\mu_{1}}=\frac{1}{\mu_{1}^{\prime}}$. Therefore $\mu_{k}^{\prime}=\mu_{k}$ for any $k \geq 1$.

The equalities $c_{k}^{\prime}=c_{k}$ follow from equation (2). It remains to show that $G^{\prime}\left(c_{k}\right)=G\left(c_{k}\right)$ for any $k \geq 1$. We do so by showing that $\left\{G\left(c_{k}\right)\right\}_{k \geq 1}$ is uniquely determined by the series $\left\{\mu_{k}\right\}_{k \geq 1}$. By equations $(3 c)$ and $(3 d), G\left(c_{k-1}\right)-G\left(c_{k}\right)=\mu_{k}$ for any $k \geq 1$. This implies that $G\left(c_{k}\right)=$ $1-\sum_{h=1}^{k} \mu_{h}$ for any $k \geq 1$. The result then follows from the equality $\mu_{k}^{\prime}=\mu_{k}$ for any $k \geq 1$ established above.

Remark A.1 We note that the condition that the firms' costs are equal (i.e., $r^{\prime}=r$ ) is adopted to ease the proof. What happens if this condition does not hold is that equation (4) implies

$$
\frac{p-r}{v-r} \sum_{k \geq 1} k \frac{\mu_{k}}{\mu_{1}}(1-F(p))^{k-1}=\frac{p-r^{\prime}}{v-r^{\prime}} \sum_{k \geq 1} k \frac{\mu_{k}^{\prime}}{\mu_{1}^{\prime}}(1-F(p))^{k-1} \quad \text { for any } p \in(\underline{p}, v],
$$


and this cannot be simplified to a power series identity as equation (A16). We however believe assuming $r^{\prime}=r$ is not necessary. Intuition suggests that the equalities $\mu_{k}^{\prime}=\mu_{k}$ and $r^{\prime}=r$ follow from (A17) as this can be viewed as a system of a continuum of equations with countably many unknowns $r, r^{\prime}, \mu_{k}, \mu_{k}^{\prime}$ for $k \geq 1$ (i.e. a system where the number of equations is significantly larger than the number of unknowns).

Lemma A.1 (Power Series) Suppose that $\left(a_{n}\right)_{n \geq 1} \subset \mathbb{R}$ and $\sum_{n \geq 1} a_{n} x^{n}=0 \forall x \in(0, \alpha)$ for some $\alpha>0$. Then $a_{n}=0$ for any $n \geq 1$.

Proof. $\sum_{n \geq 1} a_{n} x^{n}=0$ implies $a_{1}+x \sum_{n \geq 0} a_{n+2} x^{n}=0 \forall x \in(0, \alpha)$. This can also be written as $\sum_{n \geq 0} a_{n+2} x^{n}=-\frac{a_{1}}{x} \forall x \in(0, \alpha)$, which means that the power series $\sum_{n \geq 0} a_{n+2} x^{n}$ converges $\forall x \in(0, \alpha)$. Then by Lemma A.2 below there exists $\rho \in(0, \alpha)$ such that $\sum_{n \geq 0} a_{n+2} x^{n}$ is uniformly convergent on $[-\rho, \rho]$. Let $p_{1}(x)$ be its limit, where $p_{1}:[-\rho, \rho] \rightarrow \mathbb{R}$, that is, $\sum_{n \geq 0} a_{n+2} x^{n}=$ $p_{1}(x) \forall x \in[-\rho, \rho]$. Therefore

$$
a_{1}=-x p_{1}(x) \quad \forall x \in[-\rho, \rho] .
$$

The function $p_{1}$ is continuous because it is the uniform limit of a sequence of continuous functions, so $\lim _{x \rightarrow 0} p_{1}(x)=p_{1}(0)=a_{2}$. This further implies that $\lim _{x \rightarrow 0} x p_{1}(x)=0$, so based on equation (A18), for any $\varepsilon>0$ there is $\delta(\varepsilon)>0$ such that $\left|a_{1}\right|=\left|x p_{1}(x)\right|<\varepsilon$ for any $x$ with $|x|<\delta(\varepsilon)$. This implies that $a_{1}=0$.

So we have obtained that $\sum_{n \geq 2} a_{n} x^{n}=0 \forall x \in(0, \alpha)$, which implies $\sum_{n \geq 2} a_{n} x^{n-1}=0 \forall x \in$ $(0, \alpha)$. By renaming the sequence $\left(a_{n}\right)_{n \geq 2}$ as $\left(b_{n}\right)_{n \geq 1}$ with $b_{n}=a_{n+1}$ we have $\sum_{n \geq 1} b_{n} x^{n}=0$ $\forall x \in(0, \alpha)$. The arguments of the previous paragraph imply that $b_{1}=0$, that is, $a_{2}=0$. Going on this way we can show that $a_{n}=0$ for any $n \geq 1$.

The following lemma is a version of a result also known as Abel's Uniform Convergence Test.

Lemma A.2 (Abel) Suppose that the series $\sum_{n \geq 0} a_{n} x_{0}^{n}$ is convergent. Then $\forall \rho$ with $0<\rho<\left|x_{0}\right|$ the series $\sum_{n \geq 0} a_{n} x^{n}$ is uniformly convergent $\forall x \in[-\rho, \rho]$.

Proof. Let $y$ be arbitrary with $0<|y|<\left|x_{0}\right|$. First we note that the convergence of the series $\sum_{n \geq 0} a_{n} x_{0}^{n}$ implies that $\lim _{n \rightarrow \infty} a_{n} x_{0}^{n}=0$ and therefore there exists $M$ with $\left|a_{n} x_{0}^{n}\right|<M \forall n$. The sequence $b_{n}=\sum_{k=0}^{n}\left|a_{k}\right||y|^{k}$ is convergent because it is increasing and

$$
\sum_{k=0}^{n}\left|a_{k}\right||y|^{k}=\sum_{k=0}^{n}\left|a_{k}\right|\left|x_{0}\right|^{k} \frac{|y|^{k}}{\left|x_{0}\right|^{k}}<M \sum_{k=0}^{n}\left|\frac{y}{x_{0}}\right|^{k} \leq \frac{M}{1-\left|\frac{y}{x_{0}}\right|} \quad \forall n,
$$


that is, $\left(b_{n}\right)_{n}$ is bounded above. Let $b=\lim _{n \rightarrow \infty} b_{n}=\sum_{k \geq 0}\left|a_{k}\right||y|^{k}$. Then the sequence $\sum_{k \geq n+1}\left|a_{k}\right||y|^{k}=b-b_{n}$, and hence it converges to 0 .

In particular, by taking $y=\rho$ we have obtained that $\sum_{k \geq n+1}\left|a_{k}\right| \rho^{k}$ converges to 0 for arbitrary $\rho$ with $0<\rho<\left|x_{0}\right|$ and by taking $y=|x|$ we have obtained that $\sum_{k \geq 0}\left|a_{k}\right||x|^{k}$ is convergent for $\forall x \in[-\rho, \rho]$. This latter statement means that the series $\sum_{k \geq 0} a_{k} x^{k}$ is absolutely convergent and hence convergent for $\forall x \in[-\rho, \rho]$. So we can write

$$
\sup _{x \in[-\rho, \rho]}\left|\sum_{k \geq 0} a_{k} x^{k}-\sum_{k=0}^{n} a_{k} x^{k}\right|=\sup _{x \in[-\rho, \rho]}\left|\sum_{k \geq n+1} a_{k} x^{k}\right| \leq \sup _{x \in[-\rho, \rho]} \sum_{k \geq n+1}\left|a_{k}\right||x|^{k} \leq \sum_{k \geq n+1}\left|a_{k}\right| \rho^{k} .
$$

Since the right hand side goes to 0 as $n \rightarrow \infty$, we have obtained that $\sum_{k=0}^{n} a_{k} x^{k}$ converges to $\sum_{k \geq 0} a_{k} x^{k}$ uniformly for $x \in[-\rho, \rho]$. 


\section{References}

[1] Acemoglu, Daron and Robert Shimer: "Wage and Technology Dispersion," Review of Economic Studies 67, 585-607, 2000.

[2] An, Yonghong, Yingyao Hu, and Matthew Shum: "Estimating First-Price Auction Models with Unknown Number of Bidders: a Misclassification Approach," forthcoming in Journal of Econometrics, 2010.

[3] Anderson, Simon P. and Régis Renault: "Consumer Information and Firm Pricing: Negative Externalities from Improved Information," International Economic Review 41, 721-42, 2000.

[4] Bakos, Yannis : "Reducing Buyer Search Costs: Implications for Electronic Marketplaces," Management Science 43, 1976-93, 1997.

[5] Bajari, Patrick, Stephanie Houghton, and Steve Tadelis: "Bidding for Incomplete Contracts: An Empirical Analysis," Mimeo, 2007.

[6] Baye, Michael R., John Morgan, and Patrick Scholten: "Information, Search and Price Dispersion," in: Terrence Hendershott (Editor), Handbook on Economics and Information Systems, Elsevier, 2006.

[7] Bester, Helmut: "Price Commitment in Search Markets," Journal of Economic Behavior and Organization 25, 109-20, 1994.

[8] Brendstrup, Bjarne and Harry J. Paarsch: "Identification and Estimation in Sequential, Asymmetric, English Auctions," Journal of Econometrics 134, 69-94, 2006.

[9] Brynjolfsson, Eric and Michael Smith: "Frictionless Commerce? A Comparison of Internet and Conventional Retailers," Management Science 46, 563-85, 2000.

[10] Burdett, Kenneth and Kenneth L. Judd: "Equilibrium Price Dispersion," Econometrica 51, 955-69, 1983.

[11] Burdett, Kenneth and Dale T. Mortensen: "Wage Differentials, Employer Size, and Unemployment," International Economic Review 39, 257-73, 1998.

[12] Donald, Stephen G. and Harry J. Paarsch: "Piecewise Pseudo-Maximum Likelihood Estimation in Empirical Models of Auctions," International Economic Review 34, 121-48, 1993. 
[13] Fenton, Victor M. and A. Roland Gallant: "Qualitative and Asymptotic Performance of SNP Density Estimators," Journal of Econometrics 74, 77-118, 1996.

[14] Gallant, A. Ronald and Douglas W. Nychka: "Semi-Nonparametric Maximum Likelihood Estimation," Econometrica 55, 363-90, 1987.

[15] Haile, Philip, Han Hong, and Matthew Shum: "Nonparametric Tests for Common Values in First-Price Sealed-Bid Auctions," NBER Working Paper \#10105, 2003.

[16] Hoadley, Bruce: "Asymptotic Properties of Maximum Likelihood Estimators for the Independent Not Identically Distributed Case," Annals of Mathematical Statistics 42, 1977-91, 1971.

[17] Hong, Han and Matthew Shum: "Using Price Distributions to Estimate Search Costs," RAND Journal of Economics 37, 257-75, 2006.

[18] Hortaçsu, Ali and Chad Syverson: "Product Differentiation, Search Costs, and Competition in the Mutual Fund Industry: A Case Study of S\&P 500 Index Funds," Quarterly Journal of Economics 119, 403-56, 2004.

[19] Janssen, Maarten C. W. and José Luis Moraga-González: "Strategic Pricing, Consumer Search and the Number of Firms," Review of Economic Studies 71, 1089-118, 2004.

[20] Kim, Jun, Paulo Albuquerque, and Bart J. Bronnenberg: "Online Demand Under Limited Consumer Search," forthcoming at Marketing Science, 2010.

[21] van der Klaauw, Bas and Ruud H. Koning: "Testing the Normality Assumption in the Sample Selection Model with an Application to Travel Demand," Journal of Business 83 Economic Statistics 21, 31-42, 2003.

[22] Koning, Pierre, Gerard J. van den Berg, and Geert Ridder: "Semi-Nonparametric Estimation of an Equilibrium Search Model," Oxford Bulletin of Economics and Statistics 62, 327-56, 2000.

[23] Lach, Saul: "Existence and Persistence of Price Dispersion: An Empirical Analysis," Review of Economics and Statistics 84, 433-44, 2002.

[24] Lal, Rajiv and Miklos Sarvary: "When and How Is the Internet Likely to Decrease Price Competition," Marketing Science 18, 485-503, 1999. 
[25] MacMinn, Richard D.: "Search and Market Equilibrium," Journal of Political Economy 88, 308-327, 1980.

[26] Moraga-González, José Luis and Matthijs R. Wildenbeest: "Maximum Likelihood Estimation of Search Costs," European Economic Review 52, 820-48, 2008.

[27] Moraga-González, José Luis, Zsolt Sándor, and Matthijs R. Wildenbeest: "Non-Sequential Search Equilibrium with Search Cost Heterogeneity," mimeo, 2010.

[28] Morgan, Peter and Richard Manning: "Optimal Search," Econometrica 53, 923-44, 1985.

[29] Van Soest, Arthur, Marcel Das, and Xiaodong Gong: "A structural labour supply model with flexible preferences," Journal of Econometrics 107, 345-74, 2002.

[30] Stahl, Dale O.: "Oligopolistic Pricing with Sequential Consumer Search," American Economic Review 79, 700-12, 1989.

[31] Stigler, George: "The Economics of Information," Journal of Political Economy 69, 213-25, 1961.

[32] Tappata, Mariano: "Rockets and Feathers: Understanding Asymmetric Pricing," Rand Journal of Economics 44, 673-87, 2009.

[33] Varian, Hal R.: "A Model of Sales," American Economic Review 70, 651-59, 1980.

[34] Wildenbeest, Matthijs R.: "An Empirical Model of Search with Vertically Differentiated Products," Mimeo, 2009.

[35] Wolinsky, Asher: "Product Differentiation with Imperfect Information," Review of Economic Studies 51, 53-61, 1984.

[36] Wolinsky, Asher: "Procurement via Sequential Search," Journal of Political Economy 113, 785-810, 2005. 